\title{
Malignant Sertoli cell tumor in Shih Tzu dog
}

\author{
Sang-Chul Kang ${ }^{1}$, Hyoung-Seok Yang ${ }^{1}$, Ji-Youl Jung ${ }^{1}$, Eun-Hye Jung ${ }^{1}$, Hee-Chun Lee ${ }^{2}$, \\ Eui-Kyung Hwang, Jae-Hoon Kim ${ }^{1, *}$ \\ ${ }^{I}$ College of Veterinary Medicine and Veterinary Medical Research Institute, Jeju National University, \\ Jeju 690-756, Korea \\ ${ }^{2}$ College of Veterinary Medicine, Gyeongsang National University, Jinju 660-701, Korea \\ ${ }^{3}$ College of Life Science and Natural Resources, Sangji University, Wonju 220-702, Korea
}

(Accepted: November 25, 2010)

\begin{abstract}
Malignant Sertoli cell tumor was diagnosed in a 5-year-old male Shih Tzu dog. Clinical features of the dog were anorexia, urinary incontinence, constipation, anemia, alopecia, and epistaxis. The dog also had unilateral cryptorchid testis in the abdomen. Several abdominal and thoracic masses were identified on radiography. Grossly, the cryptorchid testicular mass was markedly enlarged to $8 \mathrm{~cm}$ in diameter. On cut surface, firm and well demarcated milk-white neoplastic areas were irregularly separated by white fibrous bands. Histologically, the testicular mass was diagnosed as tubular pattern Sertoli cell tumor. In addition, abdominal and mediastinal lymph nodes metastasis were found. Immunohistochemically, the tumor cells were strongly positive for vimentin and neuron specific enolase, but negative for S-100 and cytokeratin.
\end{abstract}

Keywords : cryptorchid testis, dog, immunohistochemistry, malignant Sertoli cell tumor

\section{Introduction}

According to the latest World Health Organization classification of tumors of domestic animals, tumors of testis are of four types: (1) sex-cord stromal tumors, which include both Sertoli cell tumor (SCT) and interstitial (Leydig) cell tumor, (2) germ cell tumors, which include seminoma, teratoma, embryonal carcinoma, and yolk sac carcinoma, (3) mixed germ cell-sex-cord stromal tumors (MGSCTs), and (4) other primary tumors of testicle $[5,7]$. These tumors are common in dogs and human beings. And the most common types of testicular tumors reported in dogs are seminoma, SCT and interstitial cell tumor, which occur with about equal frequency $[5,6]$.

SCTs, also called sustentacular cell tumors arise from the supporting cells of the seminiferous tubules in testis $[2,7]$. They are common in dogs, especially cryptorchid testicles, while are uncommon in other domestic animals such as stallion, ram, cat, and bull $[4,5,7]$. The metastatic rate of SCT is about $10 \sim 20 \%$, and the sites of metastasis include regional lymph nodes, kidney,

*Corresponding author

Tel: +82-64-754-3387, Fax: +82-64-702-9920

E-mail: kimjhoon@jejunu.ac.kr liver, spleen, lung, adrenal gland and pancreas [2, 8, 12]. In the present study, we described malignant SCT in a Shih Tzu dog with emphasis on the diagnostic approach including immunohistochemistry.

\section{Case Report}

A 5-year-old male Shih Tzu dog had a 1-week history of anorexia, severe anemia, urinary incontinence, constipation, alopecia, and epistaxis (Fig. 1A). The dog also had unilateral cryptorchid testis in the abdomen. On radiologic examination, various sized abdominal masses with displacement of other internal organs were noted (Fig. 1B). In the thorax, a large round mass up to $4 \mathrm{~cm}$ in diameter considered as cranial mediastinal lymph node and right displacement of the trachea due to the mass were noted. The $\operatorname{dog}$ died in the middle of preparing further examinations. Tissues samples taken at necropsy were submitted to the pathology laboratory at the College of Veterinary Medicine in Jeju National University, Korea. Large cryptorchid testis and 3 abdominal masses suspect abdominal lymph node, 

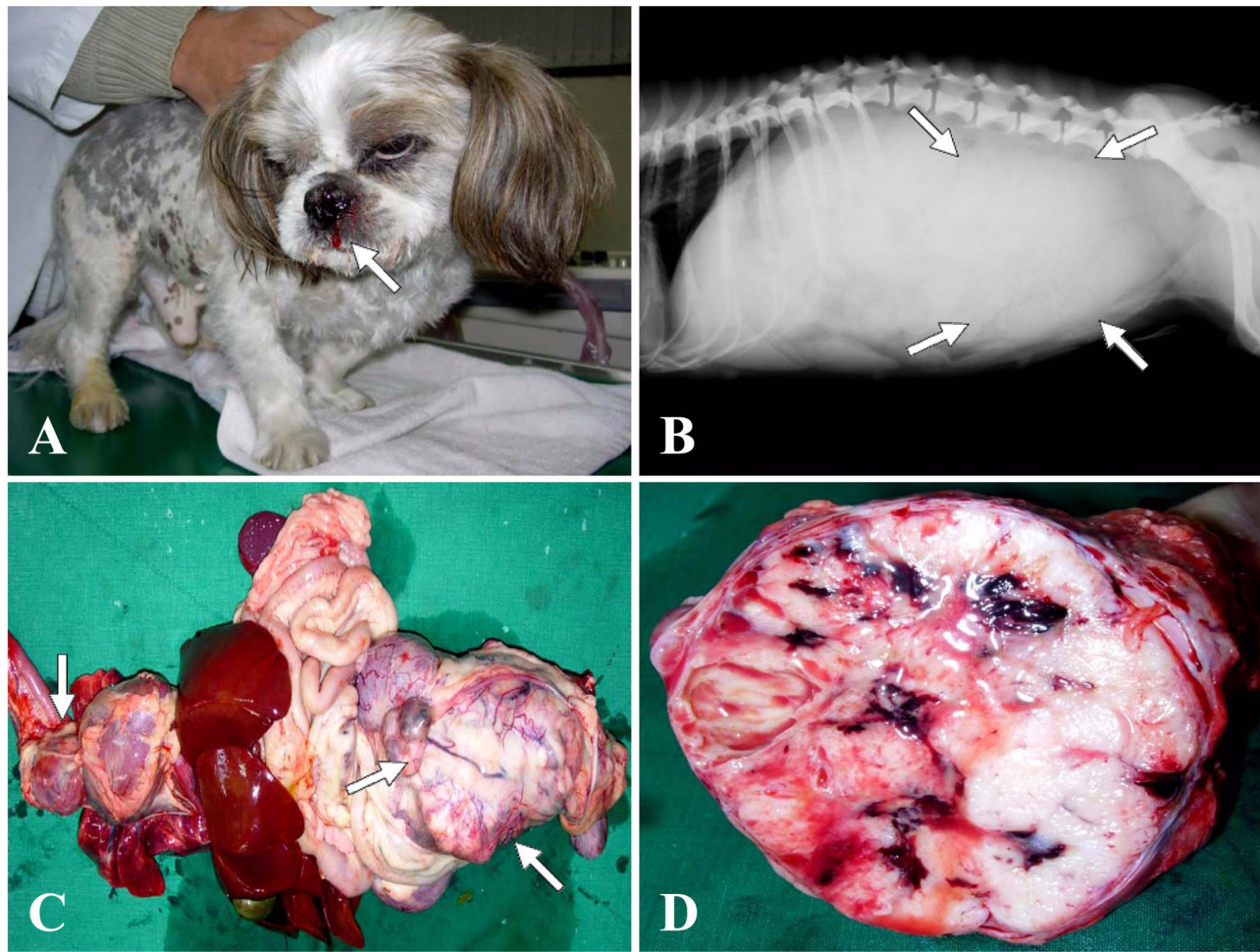

Fig. 1. Clinical signs and gross findings of malignant Sertoli cell tumor in dog. (A) Note epistaxis (arrow) and symmetrical alopecia. (B) Lateral view of radiography revealed increased soft-tissue opacity in the caudal abdomen and displacement of other organs (arrows). (C) Multiple firm, smooth, round masses about $3 \sim 8 \mathrm{~cm}$ in diameter in abdomen and thorax (arrows). (D) Cut surface of the abdominal cryptorchid mass up to $8 \mathrm{~cm}$ in diameter.

prostate, liver, kidney, spleen, and thoracic mass were fixed in $10 \%$ neutral buffered formalin for histopathologic examination. Submitted tissues were trimmed, embedded in paraffin, sectioned at $3 \mu \mathrm{m}$, and stained with hematoxylin and eosin (H\&E) for light microscopic examination. Additional paraffin-embedded sections were available for immunohistochemistry. After mounting on silane coated glass slides, each section was stained by a labeled streptavidin-biotin peroxidase method. For the differential diagnosis, primary antibody for vimentin (1: 100, monoclonal mouse anti-vimentin, clone V9; Dako, Denmark), S-100 (1:400, rabbit polyclonal anti-S100; Dako, Denmark), neuron-specific enolase (1:100, NSE, monoclonal mouse anti-human NSE, clone BBS/NC/VIH14; Dako, Denmark), and cytokeratin (1:100, monoclonal mouse anti-cytokeratin, clone AE1/AE3 and MNF 116; Dako, Denmark) were used.

At necropsy, enlarged cryptorchid testis and other small masses ranged from 2 to $4 \mathrm{~cm}$ in diameter were occupied in abdominal cavity and thorax (Fig. 1C). The cryptorchid testis was enlarged with approximately $8 \mathrm{~cm}$ in diameter. The surface was irregular and hyperemic with distended vessels. On the cut surface of testicular mass, there was loss of normal architectures due to the presence of milky white neoplastic areas irregularly separated with white fibrous bands (Fig. 1D). Multifocal hemorrhage and cavitation were observed to a variable extent throughout the testis. The prostate gland and mediastinal lymph node were severely enlarged.

Histopathologically, whole testicular parenchyma was replaced by neoplastic tubular structures. Tunica albuginea was severely thickened with fibrosis. Densely packed irregular sized neoplastic tubules were separated by a well-developed fibrovascular stroma. Tubules were composed of multilayered, moderate pleomorphic fusiform or polyhedral cells arranged perpendicular to the basement membrane with or without central necrotic cells (Fig. 2A). These tumor cells had round to elongate nuclei and either dense eosinophilic or vacuolated cytoplasm with indistinct cellular border and streaming 


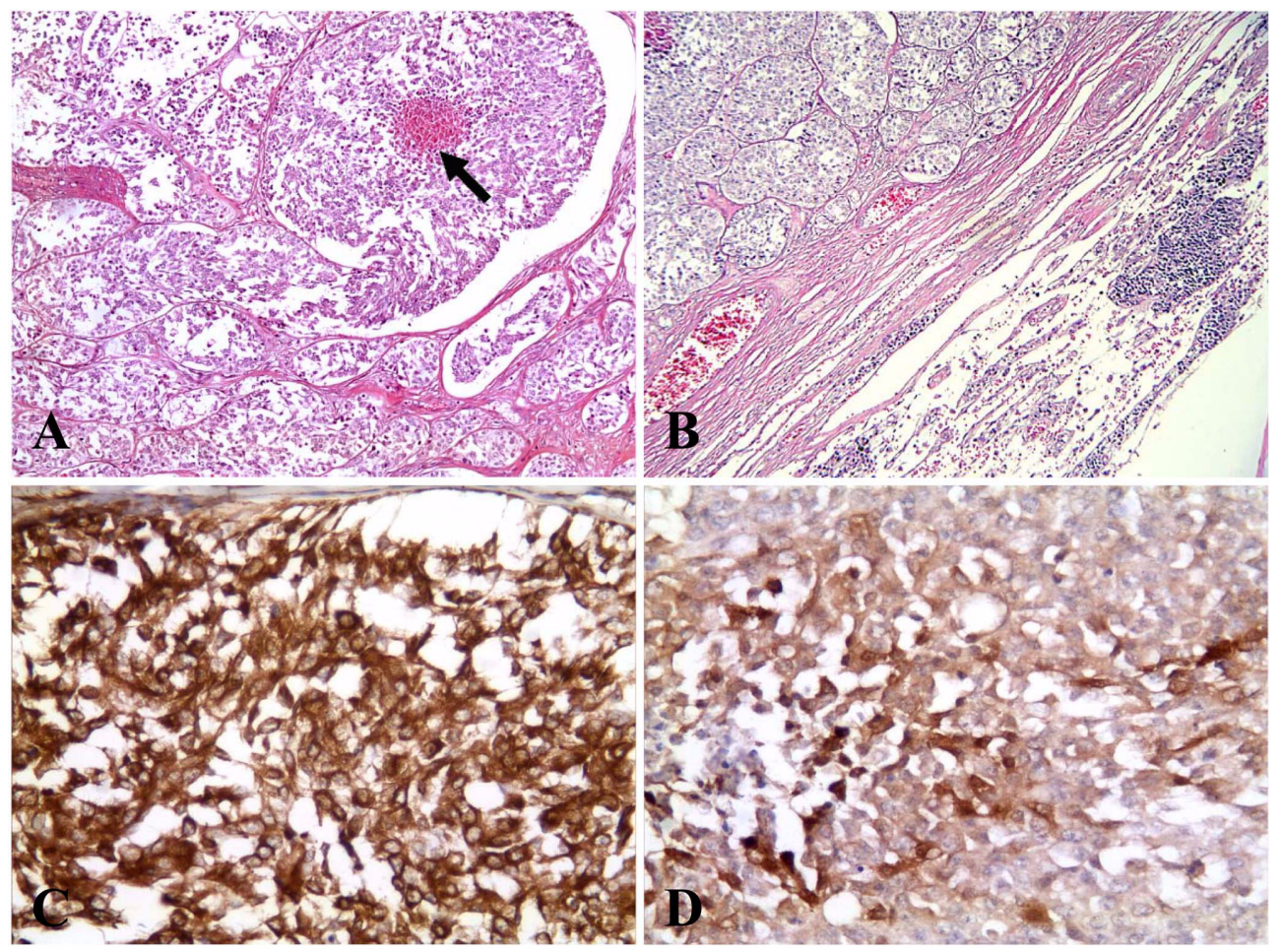

Fig. 2. Histopathologic and immunohistochemical findings of malignant Sertoli cell tumor in dog. (A) Multilobular mass of densely cellular islands are separated by fibrous septa of various widths. H\&E stain, $\times 100$. Note central necrosis (arrow). (B) Metastatic tumor in the mediastinal lymph node. H\&E stain, $\times 200$. Neoplastic tumor cells express positive reaction for vimentin (C) and neuron specific enolase (D). Streptavidin-biotin peroxidase stain, $\times 200$.

pattern. Some large neoplastic tubules contained eosinophilic central necrotic area. Many small immature tubules invaded into thickened tunica albuginea. Severe multifocal hemorrhage and necrosis also accompanied in the testicular mass. The mitotic index was 0-1 per high power field. Morphologically the tumor cells resembled Sertoli cell in testis. Histological features of metastatic foci in the mediastinal lymph node and regional abdominal lymph nodes were consistent with primary tumor lesion (Fig. 2B). Prostatic acini were transformed into stratified squamous epithelium with central cores of laminated keratin. Immunohistochemically, the neoplastic cells were strongly positive for vimentin (Fig. 2C) and NSE (Fig. 2D) expressing a diffuse cytoplasmic staining. But the neoplastic cells were negative for cytokeratin and S-100.

\section{Discussion}

Based on the gross findings, histopathology and immunohistochemistry, this case was diagnosed as a malignant SCT.

Immunohistochemical staining using a panel of antibodies have been used to confirm the histogenesis of primary testicular tumor $[1,9,10]$. In this case, the neoplastic cells stained positive for vimentin and NSE but negative for cytokeratin and S-100. According to previous study, vimentin was expressed in various degrees in all three primary testicular tumors such as seminoma, SCT, and Leydig cell tumor $[9,10]$. The only cells both in the normal testis and in the testicular tumor that stained with NSE were the Sertoli cell [10]. However, immunohistochemical staining characters of neoplastic Sertoli cells for S-100 has been varied results and it has been concluded that no generalization can be made concerning staining of neoplastic Sertoli cells for S-100 $[1,4,9]$. The results of cytokeratin stain for SCT also has been varied from different laboratories [9, 10]. In summary of immunohistochemical results, this case was confirmed as malignant SCT.

The precise differences between benign and malignant 
SCT was not well documented in veterinary literatures. In human medicine, several features including mitotic figures, pleomorphism, large tumor size and necrosis, particularly occurring together suggests malignancy of SCT [13]. Malignant tumors are large and exhibit extratesticular spread and angiolymphatic invasion. The metastatic rate is very low in small tumors less than 2 $\mathrm{cm}$ [7]. Histopathologically, SCT with diffuse pattern is more likely to be associated with malignancy, whereas the intratubular pattern usually is benign [7]. Although this case is categorized as tubular pattern, severe hemorrhagic and necrotic foci are scattered throughout the large testicular mass. In addition, the histopathologic features indicated a malignant nature of the tumor that was invasive tendency to tunica albuginea, high mitosis, and metastasis to regional and mediastinal lymph nodes.

About 20 to $30 \%$ of dogs with SCT showed signs of feminization associated with hyperestrinism, contralateral testicular atrophy, squamous metaplasia within the prostate gland, symmetrical alopecia, and bone marrow atrophy $[2,7,8,11]$. The dog, in this case, had the history of alopecia, anemia, enlarged prostate gland with histologic suqamous metaplasia, and breeding tendency. The clinical signs of anemia and epistaxis may closely be related with thrombocytopenia due to the bone marrow suppressive effects accompanying SCT [7]. However feminization was not evident in this case.

Cryptorchidism has been associated with testicular tumors in animals and human $[5,7,13]$. In dogs, cryptorchid testicles are approximately 13 to 13.6 times more likely to develop a tumor than are scrotally-located testicles. The relationship between location of cryptorchid testis and the developing tumor may be influenced by the effect of increased temperature on the testis. Increased temperatures tend to destroy spermatogenic cells, leaving SCTs free to develop [8]. Both seminoma and SCT are seen more common in cryptorchid dogs $[5,8]$. Some anaplastic SCTs containing areas resembling seminoma, often with no clear demarcation of these areas from the rest of the tumor, have been described in human and veterinary medicine $[10,13]$. MGSCTs composed of dual population of germ cell and Sertoli cells have been described in dogs [5, 9]. The incidence of testicular tumors in dog is reported to have increased during the past 40 years [3]. Therefore accurate diagnosis for testicular tumors is warranted to improve treatment and prognosis. Immunohistochemical staining combined with NSE and vimentin is useful methods for the diagnosis of testicular tumors in dog, especially in malignant SCT cases.

\section{Conclusion}

Malignant SCT was diagnosed in a 5-year-old male Shih Tzu dog with unilateral cryptorchidism. The characteristic histopathologic features such as multifocal necrotic foci, invasive growth to tunica albuginea, increased mitotic figures, and metastasis to regional lymph nodes indicated that this case was malignant. Immunohistochemical methods could be strongly supportive of the diagnosis of testicular tumors in dog, especially in SCT cases.

\section{References}

1. Doxsee AL, Yager JA, Best SJ, Foster RA. Extratesticular interstitial and Sertoli cell tumors in previously neutered dogs and cats: a report of 17 cases. Can Vet J 2006, 47, 763-766.

2. Gopinath D, Draffan D, Philbey AW, Bell R. Use of intralesional oestradiol concentration to identify a functional pulmonary metastasis of canine sertoli cell tumour. J Small Anim Pract 2009, 50, 198-200.

3. Grieco V, Riccardi E, Greppi GF, Teruzzi F, Iermanò V, Finazzi M. Canine testicular tumours: a study on 232 dogs. J Comp Path 2008, 138, 86-89.

4. Jensen KL, Krag L, Boe-Hansen GB, Jensen HE, Lehn-Jensen H. Malignant sertoli cell tumour in a young simmenthal bull - clinical and pathological observations. Reprod Domest Anim 2008, 43, 760-763.

5. Kennedy PC, Cullen JM, Edwards JF, Goldschmidt MH, Larsen S, Munson L, Nielsen S. Histological Classification of Tumors of the Genital System of Domestic Animals. Second series, Volume IV. pp. 1523, Armed Forces Institute of Pathology, Washington, 1998.

6. Ladds PW. The male genital system. In: Jubb KVF, Kennedy PC, Palmer N (eds.). Pathology of Domestic Animals. 4th ed. pp. 504-511, Academic Press, San Diego, 1997.

7. MacLachlan NJ, Kennedy PC. Tumors of the genital systems. In: Meuten DJ (ed.). Tumors in Domestic Animals. 4th ed. pp.561-567, Iowa State Press, Ames, 2002.

8. Morrison WB. Cancer in Dogs and Cats: Medical and 
Surgical Management. 2nd ed. pp. 558-560, Teton New Media, Jackson, 2002.

9. Owston MA, Ramos-Vara JA. Histologic and immunohistochemical characterization of a testicular mixed germ cell sex cord-stromal tumor and a leydig cell tumor in a dog. Vet Pathol 2007, 44, 936-943.

10. Patnaik AK, Mostofi FK. A clinicopathologic, histologic, and immunohistochemical study of mixed germ cell-stromal tumors of the testis in 16 dogs. Vet Pathol 1993, 30, 287-295.

11. Peters MAJ, de Jong FH, Teerds KJ, de Rooij DG,
Dieleman SJ, van Sluijs FJ. Ageing, testicular tumours and the pituitary-testis axis in dogs. J Endocrinol 2000, 166, 153-161.

12. Robbins M. Reproductive oncology. In: Slatter DH (ed.). Textbook of Small Animal Surgery. 3rd ed. pp. 2442-2444, Saunders, Philadelphia, 2003.

13. Young RH. Sex cord-stromal tumors of the ovary and testis: their similarities and differences with consideration of selected problems. Mod Pathol 2005, 18 (Suppl 2), S81-98. 